\title{
List of IJME reviewers in 2020
}

https://doi.org/10.2478/ijme-2020-0033

\begin{tabular}{|c|c|c|c|}
\hline Alam, Muhammad Zubair & Lahore & Pakistan & Superior University \\
\hline Ayodele, Oniku & Akoka Yaba & Nigeria & University of Lagos \\
\hline Baranowska-Prokop, Ewa & Warsaw & Poland & SGH Warsaw School of Econmics \\
\hline Bartosik-Purgat, Małgorzata & Poznan & Poland & Poznan University of Economics and Business \\
\hline Bein, Murad A. & Lefkosa & Cyprus & Uluslararasi Kibris University \\
\hline Berent, Tomasz & Warsaw & Poland & SGH Warsaw School of Economics \\
\hline Bilan, Irina & lasi & Romania & Alexandru Ioan Cuza University \\
\hline Borowski, Jakub & Warsaw & Poland & SGH Warsaw School of Economics \\
\hline Cicea, Claudiu & Bucuresti & Romania & Bucharest University of Economic Studies \\
\hline Cicek, Serkan & Fethiye & Turkey & Mugla Sitki Kocman University \\
\hline Cieślukowski, Maciej & Poznań & Poland & Poznan University of Economics and Business \\
\hline Danik, Lidia & Warsaw & Poland & SGH Warsaw School of Economics \\
\hline Demir, Volkan & İstanbul & Turkey & Galatasaray University \\
\hline Dzienis, Anna & Warsaw & Poland & SGH Warsaw School of Economics \\
\hline Dzikowska, Marlena & Poznan & Poland & Poznan University of Economics and Business \\
\hline Éltető, Andrea & Budapest & Hungary & Hungarian Academy of Sciences \\
\hline Falkowski, Krzysztof & Warsaw & Poland & SGH Warsaw School of Economics \\
\hline First-Komen, Ivana & Rijeka & Croatia & University of Rijeka \\
\hline Folfas, Pawet & Warszawa & Poland & SGH Warsaw School of Economics \\
\hline Fratocchi, Luciano & L'Aquila & Italy & University of L'Aquila \\
\hline Grudecka, Anna & Warsaw & Poland & SGH Warsaw School of Economics \\
\hline Haldia, Ashok & Ghaziabad & India & Institute of Management Technology \\
\hline Havran, Daniel & Budapest & Hungary & Corvinus University of Budapest \\
\hline $\begin{array}{l}\text { Hillemane, Bala } \\
\text { Subrahmanya Mungila }\end{array}$ & Bangalore & India & Indian Institute of Science (IISC) \\
\hline Hkiri, Besma & Jeddah & Saudi Arabia & University of Jeddah \\
\hline Holscher, Jens & Bournemouth & United Kingdom & Bournemouth University \\
\hline Hoque, Mohammad Enamul & Bangi & Malaysia & National University of Malaysia \\
\hline Hsieh, Wen-Jen & Tainan City & Taiwan & National Cheng Kung University \\
\hline Kachniewska, Magdalena & Warsaw & Poland & SGH Warsaw School of Economics \\
\hline Karbowski, Adam & Warsaw & Poland & SGH Warsaw School of Economics \\
\hline Kaya, Ayşe & Izmir & Turkey & Izmir Katip Celebi University \\
\hline Kazancoglu, Ipek & Izmir & Turkey & Ege University \\
\hline Kijek, Arkadiusz & Lublin & Poland & Maria Curie-Skłodowska University \\
\hline Konopczak, Michat & Warsaw & Poland & SGH Warsaw School of Economics \\
\hline Kowalski, Arkadiusz & Warsaw & Poland & SGH Warsaw School of Economics \\
\hline Kueh, Jerome & Kuching & Malaysia & University of Malaysia, Sarawak \\
\hline Kupczyk, Radosław & Wroctaw & Poland & University of Wroctaw \\
\hline Kuźnar, Andżelika & Warsaw & Poland & SGH Warsaw School of Economics \\
\hline
\end{tabular}




\begin{tabular}{|c|c|c|c|}
\hline Lechman, Ewa & Gdansk & Poland & Gdańsk University of Technology \\
\hline Liew, Venus Khim-Sen & Sarawak & Malaysia & University of Malaysia, Sarawak \\
\hline Liu, Weidong & Beijing & China & Chinese Academy of Sciences \\
\hline Majercakova, Daniela & Bratislava & Slovakia & Comenius University Bratislava \\
\hline Martins, Antonio & Coimbra & Portugal & University of Coimbra \\
\hline Masili, Giorgia & Roma & Italy & University of Rome Tor Vergata \\
\hline Masurasvili, loseb & Tbilisi & Georgia & New Vision University \\
\hline Matejun, Marek & Lodz & Poland & University of Lodz \\
\hline McCaleb, Agnieszka & Warsaw & Poland & SGH Warsaw School of Economics \\
\hline Ripollés Meliá, María & Castelló & Spain & Jaume I University \\
\hline Mesa, Juan Carlos Perez & Almería & Spain & University of Almería \\
\hline Mikita, Malgorzata & Warsaw & Poland & SGH Warsaw School of Economics \\
\hline Milewicz, Waldemar & Warsaw & Poland & SGH Warsaw School of Economics \\
\hline Mińska-Struzik, Ewa & Poznan & Poland & Poznan University of Economics and Business \\
\hline Mirońska, Dominika & Warsaw & Poland & SGH Warsaw School of Economics \\
\hline Miroński, Jacek & Warsaw & Poland & SGH Warsaw School of Economics \\
\hline Moisescu, Ovidiu I. & Cluj-Napoca & Romania & Babes-Bolyai University \\
\hline Morgan, George & Blacksburg & United States & Virginia Polytechnic Institute and State University \\
\hline Napiorkowski, Tomasz & Warsaw & Poland & SGH Warsaw School of Economics \\
\hline Naqvi, Syed Waqar Azeem & Montreal & Canada & McGill University \\
\hline Nasir, Nadia & Lahore & Pakistan & Superior University \\
\hline Naumovska, Ljupka & Skopje & North Macedonia & University of Tourism \& Management \\
\hline Nowak, Wioletta & Wroclaw & Poland & University of Wroclaw \\
\hline Nyga-Łukaszewska, Honorata & Warsaw & Poland & SGH Warsaw School of Economics \\
\hline Oleksiuk, Adam & Warsaw & Poland & SGH Warsaw School of Economics \\
\hline Oręziak, Leokadia & Warsaw & Poland & SGH Warsaw School of Economics \\
\hline Ostapenko, Nataliia & Tartu & Estonia & Tartu Ulikool \\
\hline Ozolina, Velga & Riga & Latvia & Riga Technical University \\
\hline Pasamehmetoglu, Aysin & Istanbul & Turkey & Özyeğin University \\
\hline Pasztorova, Janka & Bratislava & Slovakia & University of Economics in Bratislava \\
\hline Patel, Ritesh & Ahmedabad & India & Nirma University \\
\hline Pawęta, Elena & Warsaw & Poland & SGH Warsaw School of Economics \\
\hline Pelle, Anita & Szeged & Hungary & University of Szeged \\
\hline Pleśniak, Agnieszka & Warsaw & Poland & SGH Warsaw School of Economics \\
\hline Próchniak, Mariusz & Warsaw & Poland & SGH Warsaw School of Economics \\
\hline Prokop, Viktor & Pardubice & Chech Republic & University of Pardubice \\
\hline Radlo, Mariusz Jan & Warsaw & Poland & SGH Warsaw School of Economics \\
\hline Rammal, Hussain & Sydney & Australia & University of Technology Sydney \\
\hline Rehman, Mobeen Ur & Islamabad & Pakistan & $\begin{array}{l}\text { Shaheed Zulfikar Ali Bhutto Institute of Science and } \\
\text { Technology (SZABIST) }\end{array}$ \\
\hline Rzepka, Agnieszka & Lublin & Poland & Lublin University of Technology \\
\hline Salehi, Mahdi & Mashhad & $\begin{array}{l}\text { Iran (the Islamic } \\
\text { Republic of) }\end{array}$ & Ferdowsi University of Mashhad \\
\hline Sass, Magdolna & Budapest & Hungary & Hungarian Academy of Sciences Centre of Excellence \\
\hline Serwa, Dobromit & Warsaw & Poland & SGH Warsaw School of Economics \\
\hline Sethibe, Tebogo Gilbert & Johannesburg & South Africa & University of South Africa \\
\hline
\end{tabular}




\begin{tabular}{llll}
\hline Shukla, Smita & Mumbai & India & University of Mumbai \\
\hline Solangi, Yasir Ahmed & Nanjing & China & Nanjing University of Aeronautics and Astronautics \\
\hline Svetlicinii, Alexandr & Taipa & Macao & University of Macau \\
\hline Szczepaniak, Iwona & Warsaw & Poland & National Research Institute \\
\hline Sznajderska, Anna & Warsaw & Poland & SGH Warsaw School of Economics \\
\hline Szomko, Natalia & Warsaw & Poland & SGH Warsaw School of Economics \\
\hline Töngür, Ünal & Antalya & Turkey & Akdeniz University \\
\hline Tanna, Sailesh & Coventry & United Kingdom & Coventry University \\
\hline Tarczyński, Waldemar & Szczecin & Poland & University of Szczecin \\
\hline Tomaszewski, Jacek & Warsaw & Poland & SGH Warsaw School of Economics \\
\hline Trąpczyński, Piotr & Poznan & Poland & Poznan University of Economics and Business \\
\hline Tusek, Boris & Zagreb & Croatia & University of Zagreb \\
\hline Urbaniak, Maciej & Lodz & Poland & University of Lodz \\
\hline Wąsowicz-Zaborek, Elżbieta & Warsaw & Poland & SGH Warsaw School of Economics \\
\hline Wyszkowska-Kuna, Joanna & Lodz & Poland & University of Lodz \\
\hline Yilanci, Veli & Sakarya & Turkey & Sakarya University \\
\hline Zhou, Peng & Qingdao & China & China University of Petroleum \\
\hline Zmuk, Berislav & Zagreb & Croatia & University of Zagreb \\
\hline & & &
\end{tabular}

